\title{
Palladium-Catalyzed Intramolecular Hydroalkylation of Unactivated Olefins with Dialkyl Ketones
}

\author{
Xiang Wang, Tao Pei, Xiaoqing Han, and Ross A. Widenhoefer* \\ P. M. Gross Chemical Laboratory \\ Duke University \\ Durham, NC 27708-0346
}

\section{Supporting Information}

Experimental procedures, analytical and spectroscopic data for new compounds and cyclohexanones (15 pages). 


\section{Experimental}

General Methods. Reactions were performed under an atmosphere of nitrogen employing standard Schlenk techniques unless otherwise stated. NMR were obtained on a Varian spectrometer operating at $400 \mathrm{MHz}$ for ${ }^{1} \mathrm{H} \mathrm{NMR}$ and $100 \mathrm{MHz}$ for ${ }^{13} \mathrm{C} \mathrm{NMR}$ in $\mathrm{CDCl}_{3}$ unless otherwise noted. IR spectra were obtained on a Bomen MB-100 FT IR spectrometer. Gas chromatography was performed on a HP 5890 gas chromatography equipped with a $25 \mathrm{~m}$ polydimethylsiloxane capillary column. Flash column chromatography was performed employing 200-400 mesh silica gel (EM); all compounds were isolated as colorless oils unless otherwise noted. Elemental analyses were performed by Complete Analysis Laboratories (Parsippany, NJ). Thin layer chromatography (TLC) was performed on $\mathrm{SiO}_{2}$ plates eluting with a 5:1 mixture of hexanes-EtOAc unless noted otherwise. $\mathrm{CH}_{2} \mathrm{Cl}_{2}$ was distilled from $\mathrm{CaH}_{2}$ prior to use. 1,4Dioxane (Aldrich, anhydrous), DMSO (Aldrich, anhydrous) and $\mathrm{PdCl}_{2}\left(\mathrm{CH}_{3} \mathrm{CN}\right)_{2}$ (Strem) were used as received.

\section{Alkenyl Ketones}

3-Butenyl benzyl ketone (Table 1, entry 1), ${ }^{1}$ benzyl 4-pentenyl ketone (Table 1, entry 9), ${ }^{2} 3$ butenyl heptyl ketone (Table 2, entry 2), ${ }^{3}$ 3-butenyl pentyl ketone (Table 2, entry 3), 3 -butenyl phenethyl ketone (Table 2, entry 4), ${ }^{4} 1$-(4-methoxyphenyl)-5-hexen-2-one (Table 2, entry 6), ${ }^{5} 3$ butenyl cyclohexyl ketone (Table 2 , entry 7), ${ }^{6}$ methyl 5-oxo-8-nonenate (Table 2, entry 8), ${ }^{7}$ were prepared by published procedures.

1-(4-Bromophenyl)-5-hexen-2-one (Table 1, entry 2). $n$-BuLi (2.5 M in hexanes, 2.4 $\mathrm{mL}, 6.0 \mathrm{mmol})$ was added dropwise to a solution of 2-but-3-enyl-[1,3]dithiane (1.04 g, $6.0 \mathrm{mmol})$ in THF ( $25 \mathrm{~mL})$ at $-20^{\circ} \mathrm{C}$ and stirred for $2 \mathrm{~h}$. The resulting solution was treated with a solution of 1-bromomethyl-4-bromobenzene $(1.50 \mathrm{~g}, 6.0 \mathrm{mmol})$ in $\mathrm{THF}(5 \mathrm{~mL})$ at $-78{ }^{\circ} \mathrm{C}$, stirred for $10 \mathrm{~min}$ and warmed slowly to room temperature. The resulting mixture was treated with saturated aqueous $\mathrm{NH}_{4} \mathrm{Cl}$, extracted with ether, washed with brine, dried $\left(\mathrm{MgSO}_{4}\right)$, and concentrated under vacuum. The residue was filtered through a plug of silica gel and eluted with hexanes:Et $2 \mathrm{O}(50: 1)$. The 
resulting solution was concentrated under vacuum to give 2-(3-butenyl)-2-(4-bromobenzyl)-[1,3]dithiane as a pale yellow oil (1.52 $\mathrm{g})$ that was used in the next step without further purification.

A solution of crude 2-(3-butenyl)-2-(4-bromobenzyl)-[1,3]-dithiane (1.52 g) in acetonitrile (15 mL) was added to a mixture of $\mathrm{N}$-chlorosuccinamide $(3.21 \mathrm{~g}, 24.0 \mathrm{mmol})$ and silver nitrate $(4.59 \mathrm{~g}, 24.0 \mathrm{mmol})$ in acetonitrile/water $(4: 1,100 \mathrm{~mL})$ at room temperature and stirred for $30 \mathrm{~min}$. Saturated aqueous $\mathrm{Na}_{2} \mathrm{SO}_{3}$, saturated aqueous $\mathrm{Na}_{2} \mathrm{CO}_{3}$, and brine were added sequentially to the resulting white suspension. The layers were separated and the aqueous layer was extracted (dichloromethane:hexanes $=1: 1)$, dried $\left(\mathrm{MgSO}_{4}\right)$, and concentrated under vacuum. The residue was chromatographed (hexanes-Et $2 \mathrm{O}=50: 1 \rightarrow 30: 1)$ to give 1-(4-bromophenyl)-5-hexen-2-one (0.66 g, 44\%). TLC: $\mathrm{R}_{f}=0.52$. ${ }^{1} \mathrm{H}$ NMR: $\delta$ 7.46-7.43 (m, $\left.2 \mathrm{H}\right), 7.08-7.06(\mathrm{~m}, 2 \mathrm{H}), 5.76(\mathrm{tdd}$, $J=5.6,10.2,17.0 \mathrm{~Hz}, 1 \mathrm{H}), 5.03-4.95(\mathrm{~m}, 2 \mathrm{H}), 3.65(\mathrm{~s}, 2 \mathrm{H}), 2.55(\mathrm{t}, J=7.4 \mathrm{~Hz}, 2 \mathrm{H}), 2.34-2.28$ (m, $2 \mathrm{H}) .{ }^{13} \mathrm{C}\left\{{ }^{1} \mathrm{H}\right\}$ NMR: $\delta$ 207.0, 137.1, 133.4, 132.1, 131.5, 121.4, 115.8, 49.6, 41.6, 28.0. IR (neat, $\mathrm{cm}^{-1}$ ): 3077, 2976, 2917, 1897, 1713, 1640, 1590, 1487, 1405, 1358, 1262, 1186, 1107, 1070, 1012, 914, 797. Anal. calcd (found) for $\mathrm{C}_{12} \mathrm{H}_{13} \mathrm{BrO}$ : C, 56.94 (57.06); H, 5.18 (4.99).

1-(3-Trifluoromethylphenyl)-5-hexen-2-one (Table 1, entry 3). 1-(3Trifluoromethylphenyl)-5-hexen-2-one was obtained in $43 \%$ overall yield as a yellow oil from reaction of 3-trifluoro-methylphenyl acetaldehyde and 3-butenyl magnesium bromide followed by oxidation using a procedure similar to that used to synthesize 1,1-diphenyl-hex-5-en-2-one.

For 1-(3-Trifluoromethylphenyl)-5-hexen-2-ol: TLC: $\mathrm{R}_{f}=0.32 .{ }^{1} \mathrm{H}$ NMR: $\delta$ 7.507.48 (m, 2 H), 7.44-7.41 (m, 2 H), 5.84 (tdd, $J=6.4,10.4,17.0$ Hz, 1 H), 5.08-4.98 (m, 2 H), 3.89$3.83(\mathrm{~m}, 1 \mathrm{H}), 2.86(\mathrm{dd}, J=4.4,13.6,1 \mathrm{H}), 2.73(\mathrm{dd}, J=8.2,13.8 \mathrm{~Hz}, 1 \mathrm{H}), 2.31-2.13(\mathrm{~m}, 2 \mathrm{H})$, 1.72-1.55 (m, $3 \mathrm{H}) .{ }^{13} \mathrm{C}\left\{{ }^{1} \mathrm{H}\right\} \mathrm{NMR}: \delta 140.0,138.5,133.2,131.1(\mathrm{q}, J=31.7 \mathrm{~Hz}), 129.2,126.2$ $(\mathrm{q}, J=3.4 \mathrm{~Hz}), 124.5(\mathrm{q}, J=270.6 \mathrm{~Hz}), 123.6(\mathrm{q}, J=3.5 \mathrm{~Hz}), 115.4,72.3,44.0,36.3,30.4$. IR (neat, $\mathrm{cm}^{-1}$ ): 3376, 3077, 2924, 1641, 1449, 1331, 1201, 1164, 1125, 1074, 915, 798, 703. Anal. calcd. (found) for $\mathrm{C}_{13} \mathrm{H}_{15} \mathrm{~F}_{3} \mathrm{O}: \mathrm{H}, 6.19$ (6.28); C, 63.93 (63.68).

For 1-(3-Trifluoromethylphenyl)-5-hexen-2-one: TLC: $\mathrm{R}_{f}=0.54 .{ }^{1} \mathrm{H}$ NMR: $\delta 7.53$ (br d, $J=7.6 \mathrm{~Hz}, 1 \mathrm{H}), 7.47-7.43(\mathrm{~m}, 2 \mathrm{H}), 7.38$ (br d, $J=8.0 \mathrm{~Hz}, 1 \mathrm{H}), 5.77$ (tdd, $J=6.4,10.4$, 
$16.8 \mathrm{~Hz}, 1 \mathrm{H}), 5.04-4.96(\mathrm{~m}, 2 \mathrm{H}), 3.77$ (s, $2 \mathrm{H}), 2.59$ (t, J = 7.4 Hz, $2 \mathrm{H}), 2.36-2.31$ (m, $2 \mathrm{H})$. ${ }^{13} \mathrm{C}\left\{{ }^{1} \mathrm{H}\right\}$ NMR: $\delta$ 206.6, 137.0, 135.3, 133.3, $131.3(\mathrm{q}, J=32.0 \mathrm{~Hz}), 129.4,126.5(\mathrm{q}, J=3.4 \mathrm{~Hz})$, $124.4(\mathrm{q}, J=271.0 \mathrm{~Hz}), 124.2(\mathrm{q}, J=3.4 \mathrm{~Hz}), 115.8,49.7,41.8,27.9$. IR (neat, $\left.\mathrm{cm}^{-1}\right): 2918$, 1718, 1332, 1164, 1124, 1075, 914, 702. Anal. calcd. (found) for $\mathrm{C}_{13} \mathrm{H}_{13} \mathrm{~F}_{3} \mathrm{O}: \mathrm{H}, 5.41$ (5.34); C, $64.46(64.25)$.

1-(2-oxo-5-hexenyl)naphthalene (Table 1, entry 4). 1-(2-oxo-5-hexenyl)-naphthalene was obtained in $70 \%$ overall yield from reaction of naphthalen-1-yl-acetaldehyde and 3-butenyl magnesium bromide, followed by oxidation using a procedure similar to that used to synthesize 1,1diphenyl-5-hexen-2-one.

For 1-naphthalen-1-yl-5-hexen-2-ol: $\mathrm{TLC}: \mathrm{R}_{f}=0.42 .{ }^{1} \mathrm{H}$ NMR: $\delta 8.05(\mathrm{~m}, 1 \mathrm{H})$, $7.88(\mathrm{~m}, 1 \mathrm{H}), 7.77(\mathrm{~m}, 1 \mathrm{H}), 7.56-7.48(\mathrm{~m}, 2 \mathrm{H}), 7.45-7.37$ (m, $2 \mathrm{H}), 5.88(\mathrm{tdd}, J=6.8,10.4,17$ Hz, 1 H), 5.11-4.99 (m, 2 H), 4.02 (m, 1 H), 3.36 (dd, $J=4.2,13.8$ Hz, $1 \mathrm{H}), 3.08$ (dd, $J=8.6,13.8$ $\mathrm{Hz}, 1 \mathrm{H}), 2.38-2.18(\mathrm{~m}, 2 \mathrm{H}), 1.77-1.71(\mathrm{~m}, 2 \mathrm{H}), 1.58(\mathrm{~s}, 1 \mathrm{H}) .{ }^{13} \mathrm{C}\left\{{ }^{1} \mathrm{H}\right\} \mathrm{NMR}: \delta 138.7,134.8$, 134.2, 132.4, 129.1, 127.9, 127.6, 126.2, 125.9, 125.7, 124.1, 115.1, 71.7, 41.4, 36.5, 30.4. Anal. calcd. (found) for $\mathrm{C}_{16} \mathrm{H}_{18} \mathrm{O}: \mathrm{H}, 8.02$ (7.94); C, 84.91 (84.77).

For 1-(2-oxo-5-hexenyl)naphthalene: TLC: $\mathrm{R}_{f}=0.53 .{ }^{1} \mathrm{H}$ NMR: $\delta$ 7.92-7.87 (m, 2 H), $7.82(\mathrm{~d}, J=8 \mathrm{~Hz}, 1 \mathrm{H}), 7.56-7.44(\mathrm{~m}, 3 \mathrm{H}), 7.40(\mathrm{~d}, J=6.8 \mathrm{~Hz}, 1 \mathrm{H}), 5.74$ (tdd, $J=6.4,10.4$, 17.2 Hz, $1 \mathrm{H}), 5.00-4.92(\mathrm{~m}, 2 \mathrm{H}), 4.13(\mathrm{~s}, 2 \mathrm{H}), 2.53$ (t, J = 7.2 Hz, $2 \mathrm{H}), 2.32-2.27$ (m, $2 \mathrm{H})$. ${ }^{13} \mathrm{C}\left\{{ }^{1} \mathrm{H}\right\} \mathrm{NMR}: \delta 208.1,137.2,134.1,132.4,131.2,129.0,128.5,128.3,126.7,126.1,125.8$, 124.1, 115 5, 48.7, 40.8, 27.9. Anal. calcd. (found) for $\mathrm{C}_{16} \mathrm{H}_{16} \mathrm{O}: \mathrm{H}, 7.19$ (7.03); C, 85.68 (85.81).

Benzyl (Z)-3-hexenyl ketone (Table 1, entry 5). Benzyl (Z)-3-hexenyl ketone was obtained in $17 \%$ overall yield from reaction of $(Z)-4$-heptenal with benzyl magnesium chloride, followed by oxidation of employing a procedure similar to that used to synthesize 1,1-diphenylhex-5-en-2-one. TLC: $\mathrm{R}_{f}=0.72 .{ }^{1} \mathrm{H}$ NMR: $\delta$ 7.35-7.31 (m, $\left.2 \mathrm{H}\right), 7.28-7.24(\mathrm{~m}, 1 \mathrm{H}), 7.21-7.19$ (m, 2 H), 5.40-5.33 (m, 1 H), 5.26-5.19 (m, 1 H), $3.68(\mathrm{~s}, 2 \mathrm{H}), 2.49$ (t, J= 7.0 Hz, 2 H), 2.31-2.25 $(\mathrm{m}, 2 \mathrm{H}), 2.06-1.98(\mathrm{~m}, 2 \mathrm{H}), 0.93(\mathrm{t}, J=7.6 \mathrm{~Hz}, 3 \mathrm{H}) .{ }^{13} \mathrm{C}\left\{{ }^{1} \mathrm{H}\right\} \mathrm{NMR}: \delta 208.2,134.6,133.2$, 
129.7, 129.0, 127.4, 127.3, 50.6, 42.2, 21.9, 20.8, 14.6. IR (neat, $\mathrm{cm}^{-1}$ ): $3062,3028,2963,2932$, $2876,1958,1885,1713,1603,1495,1453,1359,1314,1260,1070,1030,970$. Anal. calcd (found) for $\mathrm{C}_{14} \mathrm{H}_{18} \mathrm{O}:$ C, 83.12 (83.02); $\mathrm{H}, 8.97$ (8.91).

Benzyl (E)-3-hexenyl ketone (Table 1, entry 6). Benzyl (E)-3-hexenyl ketone was synthesized in 39\% overall yield from reaction of phenylacetaldehyde and (E)-3-hexenyl magnesium bromide, followed by oxidation employing a procedure similar to that used to synthesize 1,1-diphenyl-hex-5-en-2-one.

For trans-1-phenyl-5-octen-2-ol: TLC: $\mathrm{R}_{f}=0.42 .{ }^{1} \mathrm{H}$ NMR: $\delta$ 7.34-7.30 $(\mathrm{m}, 2 \mathrm{H})$, 7.26-7.21 (m, 3 H), 5.55-5.48 (m, 1 H), 5.46-5.38 (m, 1 H), 3.87-3.81 (m, $1 \mathrm{H}), 2.83$ (dd, $J=4.4$, $13.6 \mathrm{~Hz}, 1 \mathrm{H}), 2.66$ (dd, $J=7.8,13.6 \mathrm{~Hz}, 1 \mathrm{H}), 2.24-2.07$ (m, $2 \mathrm{H}), 2.04-1.97$ (m, $2 \mathrm{H}), 1.62-1.57$ $(\mathrm{m}, 3 \mathrm{H}), 0.97(\mathrm{t}, J=7.6 \mathrm{~Hz}, 3 \mathrm{H}) .{ }^{13} \mathrm{C}\left\{{ }^{1} \mathrm{H}\right\} \mathrm{NMR}: \delta 138.9,133.1,129.8,128.8,126.8,72.6$, 44.3, 36.8, 29.3, 25.9, 14.2. IR (neat, $\mathrm{cm}^{-1}$ ): 3386, 3083, 3060, 3025, 2959, 2930, 2870, 2848, 1601, 1494, 1451, 1079, 966. Anal. calcd (found) for $\mathrm{C}_{14} \mathrm{H}_{20} \mathrm{O}$ : C, 82.30 (82.18); H, 9.87 (9.69).

For benzyl $(\boldsymbol{E})-3$-hexenyl ketone. TLC: $\mathrm{R}_{f}=0.71 .{ }^{1} \mathrm{H}$ NMR: $\delta 7.30(\mathrm{~m}, 2 \mathrm{H}), 7.26-$ $7.21(\mathrm{~m}, 1 \mathrm{H}), 7.17$ (m, $2 \mathrm{H}), 5.45-5.38(\mathrm{~m}, 1 \mathrm{H}), 5.33-5.25(\mathrm{~m}, 1 \mathrm{H}), 3.65(\mathrm{~s}, 2 \mathrm{H}), 2.48(\mathrm{t}, J=7.4$ $\mathrm{Hz}, 2 \mathrm{H}), 2.24-2.18(\mathrm{~m}, 2 \mathrm{H}), 1.97-1.89(\mathrm{~m}, 2 \mathrm{H}), 0.90(\mathrm{t}, J=7.6 \mathrm{~Hz}, 3 \mathrm{H}) .{ }^{13} \mathrm{C}\left\{{ }^{1} \mathrm{H}\right\} \mathrm{NMR}: \delta$ 208.2, 134.6, 133.4, 129.7, 129.0, 127.5, 127.3, 50.6, 42.4, 27.0, 25.8, 14.1. IR (neat, $\mathrm{cm}^{-1}$ ): 3062, 3028, 2961, 2930, 2872, 2848, 1943, 1868, 1713, 1602, 1495, 1454, 1407, 1361, 1188, 1076. Anal. calcd (found) for $\mathrm{C}_{14} \mathrm{H}_{18} \mathrm{O}: \mathrm{C}, 83.12$ (82.94); $\mathrm{H}, 8.97$ (9.02).

1,1-Diphenyl-5-hexen-2-one (Table 1, entry 7). Diphenyl acetaldehyde (2.16 g, 11.0 mmol) was added to a solution of 3-butenyl magnesium bromide (0.5 M in THF, $24.2 \mathrm{~mL}, 12.1$ $\mathrm{mmol})$ in THF $(30 \mathrm{~mL})$ at room temperature. The solution was refluxed for $12 \mathrm{~h}$ and then quenched by addition of saturated aqueous $\mathrm{NH}_{4} \mathrm{Cl}$. The resulting mixture was extracted with ether, and the combined ether extracts were washed with brine, dried $\left(\mathrm{MgSO}_{4}\right)$, and concentrated under vacuum. The resulting residue was chromatographed (hexanes-EtOAc $=40: 1$ ) to give 1,1diphenyl-5-hexen-2-ol (0.66 g, $24 \%)$. 
A solution of DMSO $(0.46 \mathrm{~mL}, 6.4 \mathrm{mmol})$ in dichloromethane $(2 \mathrm{~mL})$ was added dropwise to a solution of oxalyl chloride $(0.27 \mathrm{~mL}, 3.08 \mathrm{mmol})$ in dichloromethane $(10 \mathrm{~mL})$ at $-78{ }^{\circ} \mathrm{C}$ and stirred for $5 \mathrm{~min}$. 1,1-Diphenyl-5-hexen-2-ol (0.648 g, $2.57 \mathrm{mmol})$ was added and the resulting solution was stirred for $1.5 \mathrm{~h}$. Triethylamine $(1.79 \mathrm{~mL}, 12.8 \mathrm{mmol})$ was added, and the reaction mixture was warmed to room temperature, poured into water, and extracted with dichloromethane. The combined dichloromethane extracts were dried $\left(\mathrm{MgSO}_{4}\right)$, concentrated and chromatographed (hexanes-EtOAc $=20: 1)$ to give 1,1-diphenyl-5-hexen-2-one as a yellow oil $(0.52 \mathrm{~g}, 81 \%)$.

For 1,1-diphenyl-5-hexen-2-ol: TLC: $\mathrm{R}_{f}=0.40 .{ }^{1} \mathrm{H}$ NMR: $\delta$ 7,38-7.36 (m, $\left.2 \mathrm{H}\right)$, 7.32-7.26 (m, $6 \mathrm{H}), 7.23-7.16(\mathrm{~m}, 2 \mathrm{H}), 5.76(\mathrm{tdd}, J=6.6,10.4,17.2 \mathrm{~Hz}, 1 \mathrm{H}), 5.02-4.91(\mathrm{~m}, 2 \mathrm{H})$, 4.35 (dt, $J=2.9,8.8 \mathrm{~Hz}, 1 \mathrm{H}), 3.87(\mathrm{~d}, J=8.4 \mathrm{~Hz}, 2 \mathrm{H}), 2.32-2.25$ (m, $1 \mathrm{H}), 2.20-2.10(\mathrm{~m}, 1 \mathrm{H})$, 1.63-1.54 (m, $2 \mathrm{H}), 1.53-1.43(\mathrm{~m}, 1 \mathrm{H}) .{ }^{13} \mathrm{C}\left\{{ }^{1} \mathrm{H}\right\}$ NMR: $\delta \quad 142.3,141.4,138.4,128.8,128.6$, 128.2, 126.8, 126.5, 114.8, 73.1, 58.9, 34.2, 30.1. IR (neat, $\mathrm{cm}^{-1}$ ): 3442, 3061, 3026, 2975, 2916, 2849, 1494, 1450, 1118, 1078, 913, 702. Anal. calcd. (found) for $\mathrm{C}_{13} \mathrm{H}_{20} \mathrm{O}: \mathrm{H}, 7.99$ (7.80); C, 85.67 (85.46).

For 1,1-diphenyl-hex-5-en-2-one: TLC: $\mathrm{R}_{f}=0.57 .{ }^{1} \mathrm{H}$ NMR: $\delta$ 7.33-7.29 (m, $\left.4 \mathrm{H}\right)$, 7.26-7.21 (m, $6 \mathrm{H}), 5.75$ (tdd, $J=6.6,10.4,17.0 \mathrm{~Hz}, 1 \mathrm{H}), 5.12(\mathrm{~s}, 1 \mathrm{H}), 5.00-4.92(\mathrm{~m}, 2 \mathrm{H}), 2.64(\mathrm{t}$, $J=7.4 \mathrm{~Hz}, 2 \mathrm{H}), 2.35-2.30(\mathrm{~m}, 2 \mathrm{H}) .{ }^{13} \mathrm{C}\left\{{ }^{1} \mathrm{H}\right\} \mathrm{NMR}: \delta 207.7,138.3,136.9,128.9,128.7,127.2$, 115.4, 64.2, 41.9, 27.9. IR (neat, $\mathrm{cm}^{-1}$ ): 3061, 3026, 1717, 1598, 1494, 1451, 1277, 1079, 1032, 1000, 916, 745, 701. Anal. calcd. (found) for $\mathrm{C}_{13} \mathrm{H}_{18} \mathrm{O}: \mathrm{H}, 7.25$ (7.22); C, 86.36 (86.33).

1-(4-Fluoro-phenyl)-6-hepten-2-one (Table 1, entry 9) Was synthesized in 56\% overall yield from reaction of 4-fluorobenzyl aldehyde and 4-pentenyl magnesium bromide, followed by oxidation employing a procedure similar to that used to synthesize 1,1-diphenyl-5hexen-2-one.

For 1-(4-fluorophenyl)-6-hepten-2-ol: TLC: $R_{f}=0.38 .{ }^{1} \mathrm{H}$ NMR: $\delta$ 7.19-7.15 (m, 2 H), 7.03-6.97 (m, 2 H), 5.81 (tdd, $J=6.6,10.417 .2 \mathrm{~Hz}, 1 \mathrm{H}), 5.04-4.94$ (m, $2 \mathrm{H}), 3.82-3.76$ (m, 1 H), $7.80(\mathrm{dd}, J=4.0,13.6 \mathrm{~Hz}, 1 \mathrm{H}), 2.63(\mathrm{dd}, J=8.4,13.6 \mathrm{~Hz}, 1 \mathrm{H}), 2.11-2.06$ (m, $2 \mathrm{H}), 1.65-1.43$ $(\mathrm{m}, 5 \mathrm{H}) .{ }^{13} \mathrm{C}\left\{{ }^{1} \mathrm{H}\right\}$ NMR: $\delta 162.0(\mathrm{~d}, J=242.8 \mathrm{~Hz}), 138.9,134.5,131.1(\mathrm{~d}, J=7.6 \mathrm{~Hz}), 115.7$ 
(d, $J=20.8 \mathrm{~Hz}$ ), 115.0, 72.9, 43.5, 36.6, 34.0, 25.3. Anal. calcd. (found) for $\mathrm{C}_{13} \mathrm{H}_{15} \mathrm{FO}: \mathrm{H}, 8.23$ (8.32); C, 74.97 (75.10).

For 1-(4-fluorophenyl)-6-hepten-2-one: TLC: $R_{f}=0.44 .{ }^{1} \mathrm{H}$ NMR: $\delta$ 7.17-7.12 (m, 2 H), 7.04-6.98 (m, 2 H), 5.72 (tdd, $J=6.6,10.4,17.0 \mathrm{~Hz}, 1 \mathrm{H}), 4.99-4.93$ (m, $1 \mathrm{H}), 3.65$ (s, $2 \mathrm{H})$, $2.46(\mathrm{t}, J=7.2 \mathrm{~Hz}, 2 \mathrm{H}), 2.04-1.99(\mathrm{~m}, 2 \mathrm{H}), 1.66$ (quintet, $J=7.2 \mathrm{~Hz}, 2 \mathrm{H}) .{ }^{13} \mathrm{C}\left\{{ }^{1} \mathrm{H}\right\} \mathrm{NMR}: \delta$ 208.2, $162.3(\mathrm{~d}, J=243.9 \mathrm{~Hz}), 138.1,131.2(\mathrm{~d}, J=7.9 \mathrm{~Hz}), 130.3,115.8(\mathrm{~d}, J=21.2 \mathrm{~Hz}), 115.6$, 49.4, 41.5, 33.3, 23.0. Anal. calcd (found) for $\mathrm{C}_{13} \mathrm{H}_{15} \mathrm{FO}: \mathrm{H}, 7.33$ (7.28); C, 75.70 (75.61).

1-(4-Bromophenyl)-6-hepten-2-one (Table 1, entry 10). 1-(4-Bromophenyl)-6hepten-2-one was obtained in 33\% overall yield as a pale yellow oil from reaction of 2-(4pentenyl)-[1,3]dithiane and 1-bromomethyl-4-fluorobenzene followed by deprotection employing a procedure similar to that used to synthesize 1-(4-bromophenyl)-5-hexen-2-one. TLC: $R_{f}=0.56$. 1H NMR: $\delta$ 7.46-7.42 (m, 2 H), 7.08-7.05 (m, 2 H), 5.73 (tdd, $J=6.8,10.6,17.0$ Hz, 1 H), 5.00$4.93(\mathrm{~m}, 2 \mathrm{H}), 3.63(\mathrm{~s}, 2 \mathrm{H}), 2.46(\mathrm{t}, J=7.4 \mathrm{~Hz}, 2 \mathrm{H}), 2.04-1.99(\mathrm{~m}, 2 \mathrm{H}), 1.66$ (quintet, $J=7.2 \mathrm{~Hz}$, $2 \mathrm{H}) .{ }^{13} \mathrm{C}\left\{{ }^{1} \mathrm{H}\right\} \mathrm{NMR}: \delta 207.8,138.1,133.5,132.1,131.4,121.3,115.6,49.6,41.6,33.3,23.0$. Anal. calcd. (found) for $\mathrm{C}_{13} \mathrm{H}_{15} \mathrm{BrO}: \mathrm{H}, 5.66$ (5.65); C, 58.44 (58.59).

1-Naphthalen-2-yl-hept-6-en-2-one (Table 1, entry 11). 1-Naphthalen-2-yl-hept-6-en2-one was synthesized one in $64 \%$ overall yield from reaction of naphthalen-2-yl-acetadehyde with 4-pentenyl magnesium bromide, followed by oxidation employing a procedure similar to that used to synthesize 1,1-diphenyl-5-hexen-2-one.

For 1-naphthalen-2-yl-hept-6-en-2-ol: TLC: $R_{f}=0.34 .{ }^{1} \mathrm{H}$ NMR: $\delta$ 7.85-7.81 (m, 3 H), 7.68 (s, $1 \mathrm{H}), 7.52-7.45(\mathrm{~m}, 2 \mathrm{H}), 7.37$ (m, $1 \mathrm{H}), 5.85$ (tdd, $J=6.8,10.4,17.0 \mathrm{~Hz}, 1 \mathrm{H}), 5.08-$ 4.97 (m, 2 H), 3.95-3.89 (m, 1 H), 3.00 (dd, $J=4.0,13.6$ Hz, 1 H), 2.82 (dd, J = 8.4, 13.4 Hz, 1 H), 2.14-2.09 (m, $2 \mathrm{H}), 1.70-1.48(\mathrm{~m}, 5 \mathrm{H}) .{ }^{13} \mathrm{C}\left\{{ }^{1} \mathrm{H}\right\} \mathrm{NMR}: \delta$ 138.9, 136.3, 133.8, 132.5, 128.4, 128.1, 128.0, 127.9, 127.7, 126.3, 125.7, 114.9, 72.6, 44.5, 36.5, 33.9, 25.3. Anal. calcd. (found) for $\mathrm{C}_{17} \mathrm{H}_{20} \mathrm{O}: \mathrm{H}, 8.39$ (8.26); C, 84.96 (84.87).

For 1-Naphthalen-2-yl-hept-6-en-2-one: TLC: $\mathrm{R}_{f}=0.42 .{ }^{1} \mathrm{H}$ NMR: $\delta$ 7.85-7.80 (m, $3 \mathrm{H}), 7.68(\mathrm{~s}, 1 \mathrm{H}), 7.51-7.45(\mathrm{~m}, 2 \mathrm{H}), 7.33(\mathrm{dd}, J=2.0,8.4,1 \mathrm{H}), 5.73(\mathrm{tdd}, J=6.8,10.4,16.8 \mathrm{~Hz}$, 
$1 \mathrm{H}), 4.99-4.93$ (m, $2 \mathrm{H}), 3.85$ (s, $2 \mathrm{H}), 2.51$ (t, $J=7.4 \mathrm{~Hz}, 2 \mathrm{H}$ ), 2.04-1.99 (m, $2 \mathrm{H}$ ), 1.68 (quintet, $J$ $=7.2 \mathrm{~Hz}, 2 \mathrm{H}) .{ }^{13} \mathrm{C}\left\{{ }^{1} \mathrm{H}\right\}$ NMR: $\delta$ 208.6, 138.2, 133.9, 132.7, 132.2, 128.7, 128.4, 128.0, 127.9, 127.8, 126.5, 126.1, 115.5, 50.7, 41.5, 33.3, 23.0. IR (neat, $\mathrm{cm}^{-1}$ ): 3053, 2935, 1712, 1411, 1313, 992, 952, 904, 828, 757. Anal. calcd. (found) for $\mathrm{C}_{17} \mathrm{H}_{18} \mathrm{O}: \mathrm{H}, 7.61$ (7.79); C, 85.67 (85.76).

1-Cyclohexyl-5-hexen-2-one (Table 2, entry 5). A solution of cyclohexylacetaldehyde (3.5 g, $28 \mathrm{mmol})$ in ether $(10 \mathrm{~mL})$ was added slowly to a solution of 3-butenyl magnesium bromide (0.5 N in THF, $70 \mathrm{~mL}, 35 \mathrm{mmol}$ ) at $0{ }^{\circ} \mathrm{C}$. The resulting mixture was stirred for $1 \mathrm{~h}$, quenched by addition of $\mathrm{HCl}(1 \mathrm{~N}, 20 \mathrm{~mL})$, and extracted with ether $(2 \times 50 \mathrm{~mL})$. The combined organic fractions were dried $\left(\mathrm{MgSO}_{4}\right)$ and distilled $\left(75-78{ }^{\circ} \mathrm{C} / 60 \mathrm{mTorr}\right)$ to give 1-cyclohexyl-5-hexen-2-ol (3.2 $\mathrm{g}, 17.6 \mathrm{mmol}, 63 \%)$ that was $80 \%$ pure by GC and used in the next step without further purification. A solution of DMSO $(0.8 \mathrm{~mL}, 11.6 \mathrm{mmol})$ in $\mathrm{CH}_{2} \mathrm{Cl}_{2}(5 \mathrm{~mL})$ was added to a solution of oxalyl chloride $(1.0 \mathrm{~mL}, 11.5 \mathrm{mmol})$ in $\mathrm{CH}_{2} \mathrm{Cl}_{2}(15 \mathrm{~mL})$ at $-78{ }^{\circ} \mathrm{C}$, stirred for $5 \mathrm{~min}$. A solution of 1-cyclohexyl-5-hexen-2-ol (2.0 g, $11.0 \mathrm{mmol})$ in $\mathrm{CH}_{2} \mathrm{Cl}_{2}(5 \mathrm{~mL})$ was added slowly to the above solution and the resulting white suspension was stirred for $2 \mathrm{~h}$, quenched with water $(15 \mathrm{~mL})$, and extracted with $\mathrm{CH}_{2} \mathrm{Cl}_{2}(2 \times 40 \mathrm{~mL})$. The combined organic fractions were dried $\left(\mathrm{MgSO}_{4}\right)$, concentrated, and distilled ( $45^{\circ} \mathrm{C} / 15-20$ mTorr) to give 1-cyclohexyl-5-hexen-2-one (1.5 g, 76\%). TLC: $\mathrm{R}_{f}=0.64 .{ }^{1} \mathrm{H}$ NMR: $\delta 5.78(\mathrm{tdd}, J=6.4 .16 .8 \mathrm{~Hz}, 1 \mathrm{H}), 5.01(\mathrm{qd}, J=1.6,17.2 \mathrm{~Hz}, 1 \mathrm{H})$, $4.95(\mathrm{tdd}, J=1.2,1.6 .10 .0 \mathrm{~Hz}, 1 \mathrm{H}), 2.46(\mathrm{t}, J=7.2 \mathrm{~Hz}, 2 \mathrm{H}), 2.27-2.32(\mathrm{~m}, 2 \mathrm{H}), 2.25(\mathrm{~d}, J=6.8$ Hz, 2 H), 1.76-1.86 (m, 1 H), 1.60-1.69 (m, 5 H), 1.19-1.31 (m, 2 H), 1.06-1.17 (m, 1 H), 0.85-0.95 (m, $2 \mathrm{H}) .{ }^{13} \mathrm{C}\left\{{ }^{1} \mathrm{H}\right\}$ NMR: $\delta 210.3,137.4,115.3,50.8,42.6,34.1,33.4,27.9,26.4,26.3$. IR (neat, $\mathrm{cm}^{-1}$ ): 2922, 2850, 1713, 1448. Anal. calcd. (found) for $\mathrm{C}_{12} \mathrm{H}_{20} \mathrm{O}: \mathrm{H}, 11.18$ (11.16); C, 79.94 (79.82).

1-Chloro-7-octen-4-one (Table 2, entry 9). 1-Chloro-7-octen-4-one was synthesized in $82 \%$ yield as from reaction of 4-chlorobutyryl chloride and 3-butenyl magnesium bromide employing a procedure similar to that used to synthesize 1-phenoxy-7-octen-4-one. TLC: $\mathrm{R}_{f}=$ 0.45. ${ }^{1} \mathrm{H}$ NMR: $\delta 5.79(\mathrm{tdd}, J=6.4,10.4,13.2 \mathrm{~Hz}, 1 \mathrm{H}), 5.03(\mathrm{qd}, J=1.6,17.2 \mathrm{~Hz}, 1 \mathrm{H}), 4.96-$ $5.00(\mathrm{~m}, 1 \mathrm{H}), 3.57(\mathrm{t}, J=6.4 \mathrm{~Hz}, 2 \mathrm{H}), 2.61(\mathrm{t}, J=7.2 \mathrm{~Hz}, 2 \mathrm{H}), 2.53(\mathrm{t}, J=7.2 \mathrm{~Hz}, 2 \mathrm{H}), 2.30-$ 
2.36 (m, $2 \mathrm{H}), 2.01-2.07$ (m, $2 \mathrm{H}) .{ }^{13} \mathrm{C}\left\{{ }^{1} \mathrm{H}\right\}$ NMR: $\delta$ 209.1, 137.1, 115.6, 44.7, 42.1, 39.5, 27.9, 26.4. IR (neat, $\mathrm{cm}^{-1}$ ): 3078, 2961, 2922, 1716, 1640, 1411. Anal. calcd. (found) for $\mathrm{C}_{8} \mathrm{H}_{13} \mathrm{ClO}$ : H, 8.16 (8.11); C, 59.81 (59.74).

1-Phenoxy-7-octen-4-one (Table 2, entry 10). A solution of 3-butenyl magnesium bromide ( $0.5 \mathrm{~N}$ in THF, $9 \mathrm{~mL}$ ) was added over $15 \mathrm{~min}$ to a suspension of $\mathrm{CuI}(0.9 \mathrm{~g}, 4.7 \mathrm{mmol})$, and 4-phenoxybutyryl chloride $(1.0 \mathrm{~g}, 5.0 \mathrm{mmol})$ in THF $(15 \mathrm{~mL})$ at $-78^{\circ} \mathrm{C}$. The resulting mixture was warmed slowly to room temperature, quenched with $\mathrm{HCl}(1 \mathrm{~N}, 20 \mathrm{~mL})$, and extracted with ether $(3 \times 30 \mathrm{~mL})$. The combined organic fractions were dried $\left(\mathrm{MgSO}_{4}\right)$, concentrated, and chromatographed (hexanes-ether $=20: 1 \rightarrow 5: 1)$ to give 1-phenoxy-6-hepten-3-one $(0.89 \mathrm{~g}, 4.1$ mmol, 91\%). TLC: $\mathrm{R}_{f}=0.40 .{ }^{1} \mathrm{H}$ NMR (300 MHz): $\delta$ 7.23-7.27 (m, $\left.2 \mathrm{H}\right), 6.85-6.84(\mathrm{~m}, 3 \mathrm{H})$, 5.79 (tdd, $J=6.6,10.2,17.1 \mathrm{~Hz}, 1 \mathrm{H}), 4.94-5.05(\mathrm{~m}, 2 \mathrm{H}), 3.96$ (t, $J=6.0 \mathrm{~Hz}, 2 \mathrm{H}), 2.62$ (t, $J=6.9$ $\mathrm{Hz}, 2 \mathrm{H}), 2.53(\mathrm{t}, J=7.5 \mathrm{~Hz}, 2 \mathrm{H}), 2.32(1, J=6.9 \mathrm{~Hz}, 2 \mathrm{H}), 2.05$ (quintet, $J=6.9 \mathrm{~Hz}, 2 \mathrm{H}$ ). ${ }^{13} \mathrm{C}\left\{{ }^{1} \mathrm{H}\right\} \mathrm{NMR}(75 \mathrm{MHz}): \delta 208.9,158.1,136.4,128.7,120.0,114.6,113.7,66.0,41.2,38.4$, 27.1, 22.7. IR (neat, $\mathrm{cm}^{-1}$ ): $3073,3039,2935,1713,1496$. Anal. calcd. (found) for $\mathrm{C}_{14} \mathrm{H}_{18} \mathrm{O}_{2}$ : H, 8.31 (8.36); C, 77.03 (76.95).

1-Methoxy-7-octen-4-one (Table 2, entry 11). 1-Methoxy-7-octen-4-one was synthesized in $28 \%$ overall yield from reaction of 4-methoxy butyraldehyde and 3-butenyl magnesium bromide, followed by oxidation employing a procedure similar to that used to synthesize 1,1-diphenyl-5-hexen-2-one.

For 1-Methoxy-7-octen-4-ol: TLC: $\mathrm{R}_{f}=0.23 .{ }^{1} \mathrm{H}$ NMR: $\delta 5.81$ (tdd, $J=6.8,10.4$, $17.2 \mathrm{~Hz}, 1 \mathrm{H}), 5.01(\mathrm{qd}, J=1.6,17.2 \mathrm{~Hz}, 1 \mathrm{H}), 4.93(\mathrm{tdd}, J=1.2,2.0,10.0 \mathrm{~Hz}, 1 \mathrm{H}), 3.55-3.61(\mathrm{~m}$, $1 \mathrm{H}), 3.75-3.41$ (m, $2 \mathrm{H}), 3.32$ (s, $3 \mathrm{H}), 2.66$ (s, $1 \mathrm{H}), 2.05-2.23$ (m, $2 \mathrm{H}), 1.38-1.71$ (m, $6 \mathrm{H})$. ${ }^{13} \mathrm{C}\left\{{ }^{1} \mathrm{H}\right\}$ NMR: $\delta$ 138.9, 114.8, 73.2, 71.1, 58.7, 36.8, 35.0, 30.3, 26.4. IR (neat, $\mathrm{cm}^{-1}$ ): 3411, 2931, 2250, 907. Anal. calcd (found) for $\mathrm{C}_{9} \mathrm{H}_{18} \mathrm{O}_{2}: \mathrm{H}, 11.47$ (11.29); C, 68.31 (68.32).

For 1-Methoxy-7-octen-4-one: TLC: $\mathrm{R}_{f}=0.25 .{ }^{1} \mathrm{H}$ NMR: $\delta 5.78(\operatorname{tdd}, J=6.4,10.0$, $17.2 \mathrm{~Hz}, 1 \mathrm{H}), 5.00$ (qd, $J=1.6,17.2 \mathrm{~Hz}, 1 \mathrm{H}), 4.95$ (tdd, $J=1.2,2.0,10.0 \mathrm{~Hz}, 1 \mathrm{H}), 3.35$ (t, $J=6.4$ Hz, 2 H), 3.28 (s, 3 H), 2.46-2.51 (m, 4 H), 2.28-2.33 (m, 2 H), 1.82 (tt, $J=6.0,7.2$ Hz, 2 H). 
${ }^{13} \mathrm{C}\left\{{ }^{1} \mathrm{H}\right\}$ NMR: $\delta 210.3,137.3,115.3,71.9,58.7,42.0,39.5,28.0,23.9 . \quad$ IR (neat, $\mathrm{cm}^{-1}$ ): 3078 , 2977, 2927, 2828, 1713, 1119. Anal. calcd. (found) for $\mathrm{C}_{9} \mathrm{H}_{16} \mathrm{O}_{2}: \mathrm{H}, 10.32(10.18)$; C, 69.19 (69.31).

\section{Cyclohexanones}

2-Phenylcyclohexanone (Table 1, entry 1). ${ }^{8}$ A suspension of benzyl 3-butenyl ketone (87 mg, $0.50 \mathrm{mmol})$, dichlorodimethylsilane (0.19 mL, $1.50 \mathrm{mmol}), \mathrm{PdCl}_{2}\left(\mathrm{MeCN}_{2}(13 \mathrm{mg}, 0.05\right.$ $\mathrm{mmol})$, and $\mathrm{CuCl}_{2}(67 \mathrm{mg}, 0.50 \mathrm{mmol})$ in 1,4-dioxane $(10 \mathrm{~mL})$ was stirred at $70{ }^{\circ} \mathrm{C}$ for $18 \mathrm{~h}$. The reaction mixture was cooled to room temperature, filtered through a plug of silica gel, and eluted with ether. The resulting solution was concentrated and the residue was chromatographed (hexanes-ether $=25: 1 \rightarrow 15: 1)$ to give 2-phenylcyclohexanone $(68 \mathrm{mg}, 78 \%)$ as a pale yellow solid. ${ }^{1} \mathrm{H}$ NMR: $\delta$ 7.33-7.29 (m, $\left.2 \mathrm{H}\right), 7.25-7.21(\mathrm{~m}, 1 \mathrm{H}), 7.13-7.11(\mathrm{~m}, 2 \mathrm{H}), 3.59(\mathrm{dd}, J=5.4,12.2$ Hz, 1 H), 2.54-2.43 (m, 2 H), 2.28-2.22 (m, 1 H), 2.16-2.10 (m, 1 H), 2.04-1.95 (m, 2 H), 1.83-1.77 (m, $2 \mathrm{H}) .{ }^{13} \mathrm{C}\left\{{ }^{1} \mathrm{H}\right\}$ NMR: $\delta$ 210.6, 139.1, 128.8, 128.7, 127.2, 57.7, 42.5, 35.4, 28.1, 25.6.

A similar procedure was employed for the synthesis of the remaining cyclohexanones found in Table 1, with the exception that $\mathrm{SiMe}_{3} \mathrm{Cl}$ was employed in place of $\mathrm{SiMe}_{2} \mathrm{Cl}_{2}$.

2-(4-Bromophenyl)cyclohexanone (Table 1, entry 2). ${ }^{9}{ }^{1} \mathrm{H}$ NMR: $\delta$ 7.47-7.44 (m, 2 H), 7.03-7.00 (m, 2 H), 3.57 (dd, $J=5.2,12.4 \mathrm{~Hz}, 1 \mathrm{H}), 2.56-2.54(\mathrm{~m}, 2 \mathrm{H}), 2.29-2.23(\mathrm{~m}, 1 \mathrm{H})$, 2.19-2.14 (m, $1 \mathrm{H}), 2.02-1.95(\mathrm{~m}, 2 \mathrm{H}), 1.87-1.78(\mathrm{~m}, 2 \mathrm{H}) .{ }^{13} \mathrm{C}\left\{{ }^{1} \mathrm{H}\right\} \mathrm{NMR}: \delta 210.0,138.1$, $131.8,130.6,121.2,57.2,42.5,35.5,28.1,25.7$.

2-(3-trifluoromethylphenyl)cyclohexanone. Yellow oil. TLC: $\mathrm{R}_{f}=0.33$. ${ }^{1} \mathrm{H}$ NMR: $\delta$ 7.52 (br d, $J=7.6 \mathrm{~Hz}, 1 \mathrm{H}), 7.48$ (br t, $J=7.6 \mathrm{~Hz}, 1 \mathrm{H}), 7.40$ (s, $1 \mathrm{H}), 7.33$ (br d, $J=8.0 \mathrm{~Hz}, 1 \mathrm{H}$ ), $3.68(\mathrm{dd}, J=5.6,12.4 \mathrm{~Hz}, 1 \mathrm{H}), 2.57-2.44$ (m, $2 \mathrm{H}), 2.32-2.27$ (m, $1 \mathrm{H}), 2.22-2.16$ (m, $1 \mathrm{H}), 2.06-$ $1.96(\mathrm{~m}, 2 \mathrm{H}), 1.89-1.78(\mathrm{~m}, 2 \mathrm{H}) .{ }^{13} \mathrm{C}\left\{{ }^{1} \mathrm{H}\right\} \mathrm{NMR}: \delta 209.7,140.0,132.5,130.9(\mathrm{q}, J=32 \mathrm{~Hz})$, 129.0, 125.7 (q, $J=3.4 \mathrm{~Hz}), 124.5$ (q, $J=208 \mathrm{~Hz}), 124.8$ (q, $J=34 \mathrm{~Hz}), 57.5$, 42.5, 35.6, 28.1, 25.7. IR (neat, $\mathrm{cm}^{-1}$ ): 2940, 2864, 1711, 1449, 1330, 1163, 1124, 1074, 799, 702. Anal. calcd. (found) for $\mathrm{C}_{13} \mathrm{H}_{13} \mathrm{O}$ : C, 64.46 (64.29); H, 5.41 (5.25). 
2-Naphthalen-1-yl-cyclohexanone (Table 1, entry 4). ${ }^{10}{ }^{1} \mathrm{H}$ NMR: $\delta$ 7.89-7.86 (m, 1 $\mathrm{H}), 7.80(\mathrm{~d}, J=8.0 \mathrm{~Hz}, 1 \mathrm{H}), 7.75-7.73(\mathrm{~m}, 1 \mathrm{H}), 7.51-7.45(\mathrm{~m}, 3 \mathrm{H}), 7.38(\mathrm{~d}, J=7.2 \mathrm{~Hz}, 1 \mathrm{H})$, 4.37 (dd, $J=5.2,12.4$ Hz, 1 H), 2.71-2.62 (m, 2 H), 2.46-2.39 (m, 1 H), 2.34-2.24 (m, 2 H), 2.17$2.11(\mathrm{~m}, 1 \mathrm{H}), 2.01-1.86(\mathrm{~m}, 2 \mathrm{H}) .{ }^{13} \mathrm{C}\left\{{ }^{1} \mathrm{H}\right\} \mathrm{NMR}: \delta 210.2,135.5,134.1,132.0,129.3,127.9$, $126.1,125.60,125.57,125.54,123.5,53.6,42.9,34.5,28.1,26.2$.

trans-3-Ethyl-2-phenylcyclohexanone (Table 1, entry 5, 6). TLC: $\mathrm{R}_{f}=0.47 .{ }^{1} \mathrm{H}$ NMR: $\delta$ 7.36-7.32 (m, $2 \mathrm{H}), 7.28-7.24(\mathrm{~m}, 1 \mathrm{H}), 7.08-7.06(\mathrm{~m}, 2 \mathrm{H}), 3.31(\mathrm{~d}, J=11.6 \mathrm{~Hz}, 1 \mathrm{H})$, 2.57-2.55 (m, $1 \mathrm{H}), 2.53-2.51(\mathrm{~m}, 1 \mathrm{H}), 2.44$ (dt, $J=6.0,13.6 \mathrm{~Hz}, 1 \mathrm{H}), 2.22-2.09$ (m, $2 \mathrm{H}), 2.03-$ $1.93(\mathrm{~m}, 1 \mathrm{H}), 1.86-1.74(\mathrm{~m}, 1 \mathrm{H}), 1.27(\mathrm{dqd}, J=3.2,7.6,13.6 \mathrm{~Hz}, 1 \mathrm{H}), 1.08(\mathrm{~m}, 1 \mathrm{H}), 0.81(\mathrm{t}, J=$ $7.6 \mathrm{~Hz}, 3 \mathrm{H}) .{ }^{13} \mathrm{C}\left\{{ }^{1} \mathrm{H}\right\} \mathrm{NMR}: \delta 210.6,137.8,129.7,128.5,127.1,63.6,46.7,42.3,30.4,27.4$, 26.2, 10.9. IR (neat, $\mathrm{cm}^{-1}$ ): 3087, 3061, 3028, 2961, 2935, 2863, 1947, 1870, 1812, 1713, 1603, $1497,1454,1445,1381,1358,1344,1310,1280,1176,1123$. Anal. calcd (found) for $\mathrm{C}_{14} \mathrm{H}_{18} \mathrm{O}$ : C, 83.12 (83.05); H, 8.97 (8.92).

2,2-Diphenylcyclohexanone (Table 1, entry 7). ${ }^{11}{ }^{1} \mathrm{H}$ NMR: $\delta \quad 7.32-7.27$ (m, $\left.4 \mathrm{H}\right)$, 7.25-7.21 (m, 2 H), 7.05-7.02 (m, 4 H), 2.58 (t, $J=5.8$ Hz, 2 H), 2.49 (t, J = 6.8 Hz, 2 H), 1.96$1.90(\mathrm{~m}, 2 \mathrm{H}), 1.85-1.79(\mathrm{~m}, 2 \mathrm{H}) .{ }^{13} \mathrm{C}\left\{{ }^{1} \mathrm{H}\right\} \mathrm{NMR}: \delta 211.3,142.3,128.5,128.3,126.8,63.9$, $40.7,39.2,27.8,22.1$.

trans-3-Methyl-2-phenylcyclohexanone (Table 1, entry 8). ${ }^{12}{ }^{1} \mathrm{H}$ NMR: $\delta$ 7.32-7.28 (m, $2 \mathrm{H}), 7.24-7.21(\mathrm{~m}, 1 \mathrm{H}), 7.05-7.03(\mathrm{~m}, 2 \mathrm{H}), 3.16(\mathrm{~d}, J=11.6 \mathrm{~Hz}, 1 \mathrm{H}), 2.53-2.48(\mathrm{~m}, 1 \mathrm{H})$, $2.41(\mathrm{dt}, J=5.6,13.6 \mathrm{~Hz}, 1 \mathrm{H}), 2.15-2.05(\mathrm{~m}, 2 \mathrm{H}), 2.03-1.96(\mathrm{~m}, 1 \mathrm{H}), 1.80(\mathrm{tq}, J=4.0,13.2 \mathrm{~Hz}, 1$ $\mathrm{H}), 1.61-1.51(\mathrm{~m}, 1 \mathrm{H}), 0.79(\mathrm{~d}, J=6.4 \mathrm{~Hz}, 3 \mathrm{H}) .{ }^{13} \mathrm{C}\left\{{ }^{1} \mathrm{H}\right\} \mathrm{NMR}: \delta 210.3,137.8,129.7,128.6$, $127.1,65.5,42.1,41.0,34.7,26.3,21.6$.

trans-2-(4-Fluorophenyl)-3-methylcyclohexanone (Table 1, entry 9). White solid. TLC: $\mathrm{R}_{f}=0.43 .{ }^{1} \mathrm{H}$ NMR: $\delta 7.03(\mathrm{~s}, 2 \mathrm{H}), 7.02(\mathrm{~s}, 2 \mathrm{H}), 3.20(\mathrm{~d}, J=11.6 \mathrm{~Hz}, 1 \mathrm{H}), 2.55-2.40$ (m, $2 \mathrm{H}), 2.18-2.00(\mathrm{~m}, 3 \mathrm{H}), 1.87-1.75(\mathrm{~m}, 1 \mathrm{H}), 1.64-1.53(\mathrm{~m}, 1 \mathrm{H}), 0.81$ (d, J = 6.4 Hz, $3 \mathrm{H})$. ${ }^{13} \mathrm{C}\left\{{ }^{1} \mathrm{H}\right\} \mathrm{NMR}: \delta 210.1,162.1(\mathrm{~d}, J=243.2 \mathrm{~Hz}), 133.4,131.0(\mathrm{~d}, J=7.9 \mathrm{~Hz}), 115.4(\mathrm{~d}, J=20.9$ 
Hz), 64.7, 42.1, 41.3, 34.6, 26.3, 21.5. IR (neat, $\mathrm{cm}^{-1}$ ): 2922, 2863, 1705, 1509, 1220, 1160, 1047, 807. Anal. calcd. (found) for $\mathrm{C}_{13} \mathrm{H}_{15} \mathrm{FO}: \mathrm{H}, 7.33$ (7.16); C, 75.70 (75.70).

trans-2-(4-Bromophenyl)-3-methylcyclohexanone (Table 1, entry 10). White solid. mp 91-92 ${ }^{\circ} \mathrm{C}$. TLC: $\mathrm{R}_{f}=0.42 .{ }^{1} \mathrm{H}$ NMR: $\delta$ 7.47-7.44 (m, $\left.2 \mathrm{H}\right), 6.96-6.93(\mathrm{~m}, 2 \mathrm{H}), 3.16(\mathrm{~d}, J=$ 11.6 Hz, $1 \mathrm{H}), 2.55-2.39$ (m, $2 \mathrm{H}), 2.18-2.00$ (m, $3 \mathrm{H}), 1.87-1.75$ (m, $1 \mathrm{H}), 1.63-1.53$ (m, $1 \mathrm{H}), 0.81$ $(\mathrm{d}, J=6.4 \mathrm{~Hz}, 3 \mathrm{H}) .{ }^{13} \mathrm{C}\left\{{ }^{1} \mathrm{H}\right\}$ NMR: $\delta 209.8,136.8,131.7,131.4,121.1,64.9,42.1,41.1,34.6$, 26.3, 21.5. IR (neat, $\mathrm{cm}^{-1}$ ): 2955, 2921, 1707, 1320, 1046, 708. Anal. calcd. (found) for $\mathrm{C}_{13} \mathrm{H}_{15} \mathrm{BrO}: \mathrm{H}, 5.66$ (5.58); C, 58.44 (58.40).

trans-3-Methyl-2-naphthalen-2-yl-cyclohexanone (Table 1, entry 11). White solid. mp 87-89 ${ }^{\circ} \mathrm{C} . \mathrm{TLC}: \mathrm{R}_{f}=0.42 .{ }^{1} \mathrm{H}$ NMR: $\delta$ 7.83-7.77 (m, $\left.3 \mathrm{H}\right), 7.52(\mathrm{~s}, 1 \mathrm{H}), 7.47-7.41(\mathrm{~m}, 2$ H), $7.22(\mathrm{dd}, J=1.2,8.4 \mathrm{~Hz}, 1 \mathrm{H}), 3.35(\mathrm{~d}, J=12.0 \mathrm{~Hz}, 1 \mathrm{H}), 2.57-2.42(\mathrm{~m}, 2 \mathrm{H}), 2.28(\mathrm{~m}, 2 \mathrm{H})$, 2.06-2.01 (m, $1 \mathrm{H}), 1.91-1.79(\mathrm{~m}, 1 \mathrm{H}), 1.66-1.56(\mathrm{~m}, 1 \mathrm{H}) .0 .82(\mathrm{~d}, J=6.4 \mathrm{~Hz}, 3 \mathrm{H}) .{ }^{13} \mathrm{C}\left\{{ }^{1} \mathrm{H}\right\}$ NMR: $\delta 210.5,135.5,133.7,132.9,128.7,128.1,128.0,127.4,126.1,125.8,65.6,42.2,40.8$, 34.6, 26.3, 21.7. IR (neat, $\mathrm{cm}^{-1}$ ): 3053, 2952, 29.25, 28.66, 1710, 1599, 1508, 1455, 1374, 1318, 1267, 1184, 1121, 1048, 962, 907, 851, 811, 745. Anal. calcd. (found) for $\mathrm{C}_{17} \mathrm{H}_{18} \mathrm{O}: \mathrm{H}, 7.61$ (7.85); C, 85.67 (85.44).

2-Hexylcyclohexanone (Table 2, entry 2). ${ }^{13}$ A suspension of $\mathrm{PdCl}_{2}\left(\mathrm{MeCN}_{2}(13 \mathrm{mg}\right.$, $0.05 \mathrm{mmol}), \mathrm{CuCl}_{2}(20 \mathrm{mg}, 0.15 \mathrm{mmol}), \mathrm{HCl}(4 \mathrm{~N}$ in dioxane, $13 \mu \mathrm{L}, 0.05 \mathrm{mmol})$, and 3-butenyl heptyl ketone $(91 \mathrm{mg}, 0.50 \mathrm{mmol})$ in dioxane $(6 \mathrm{~mL})$ was heated at $70{ }^{\circ} \mathrm{C}$ for $12 \mathrm{~h}$ in a sealed tube. The resulting mixture was filtered through a short plug of silica gel, eluting with hexanes/ether (1:1, $30 \mathrm{~mL}$ ). The resulting solution was concentrated and chromatographed (hexanes-ether $=20: 1$

$\rightarrow 10: 1)$ to give 2-hexylcyclohexanone (70 $\mathrm{mg}, 77 \%$ ) as a pale yellow oil. ${ }^{1} \mathrm{H}$ NMR: $\delta$ 2.33-2.38 (m, 1 H), 2.20-2.30 (m, 2 H), 1.96-2.11 (m, 2 H), 1.57-1.85 (m, 2 H), 1.30-1.39 (m, 1 H), 1.13-1.29 $(\mathrm{m}, 9 \mathrm{H}), 0.83(\mathrm{t}, J=6.8 \mathrm{~Hz}, 3 \mathrm{H}) .{ }^{13} \mathrm{C}\left\{{ }^{1} \mathrm{H}\right\} \mathrm{NMR}: \delta \quad 213.8,50.9,42.1,34.0,31.9,29.6,29.6$, $28.2,27.3,25.0,22.8,14.2$.

A similar procedure was employed for the synthesis of the remaining cyclohexanones found in Table 2. 
2-Butylcyclohexanone (Table 2, entry 3). ${ }^{14}{ }^{1} \mathrm{H}$ NMR: $\delta \quad 2.34-2.40(\mathrm{~m}, 1 \mathrm{H}), 2.21$ 2.31 (m, 2 H), 2.08-2.12 (m, 1 H), 2.00-2.03 (m, 1 H), 1.61-1.87 (m, 4 H), 0.90-1.60 (m, 6 H), 0.88 (t, $J=7.2 \mathrm{~Hz}, 3 \mathrm{H}) .{ }^{13} \mathrm{C}\left\{{ }^{1} \mathrm{H}\right\}$ NMR: $\delta 213.8,50.9,42.1,34.0,29.6,29.3,28.2,25.0,23.0,14.2$.

2-Benzylcyclohexanone (Table 2, entry 4). ${ }^{15}{ }^{1} \mathrm{H}$ NMR: $\delta \quad 7.25(\mathrm{t}, J=7.2 \mathrm{~Hz}, 2 \mathrm{H})$, $7.17(\mathrm{~d}, J=6.8 \mathrm{~Hz}, 1 \mathrm{H}), 7.13(\mathrm{~d}, J=7.6 \mathrm{~Hz}, 2 \mathrm{H}), 3.21(\mathrm{dd}, J=6.4,11.2 \mathrm{~Hz}, 1 \mathrm{H}), 2.49-2.57(\mathrm{~m}, 1$ H), 1.49-1.71 (m, $2 \mathrm{H}), 1.33(\mathrm{dq}, J=3.6,12.4 \mathrm{~Hz}, 1 \mathrm{H}) .{ }^{13} \mathrm{C}\left\{{ }^{1} \mathrm{H}\right\} \mathrm{NMR}: \delta 212.7,140.6,129.3$, $128.5,126.2,52.7,42.4,35.7,33.6,28.3,25.3$.

2-Cyclohexylcyclohexanone (Table 2, entry 5). ${ }^{16}{ }^{1} \mathrm{H}$ NMR: $\delta \quad 2.30-2.36(\mathrm{~m}, 1 \mathrm{H})$, 2.18-2.26 (m, $1 \mathrm{H}), 2.02-2.08(\mathrm{~m}, 1 \mathrm{H}), 1.86-1.94(\mathrm{~m}, 2 \mathrm{H}), 1.47-1.83(\mathrm{~m}, 10 \mathrm{H}), 1.24(\mathrm{tq}, J=3.2$, $12.8 \mathrm{~Hz}, 2 \mathrm{H}), 1.09$ (tq, $J=2.2,12.4 \mathrm{~Hz}, 1 \mathrm{H}), 0.96(\mathrm{dq}, J=3.2,12.0 \mathrm{~Hz}, 1 \mathrm{H}), 0.80-0.90(\mathrm{~m}, 1 \mathrm{H})$. ${ }^{13} \mathrm{C}\left\{{ }^{1} \mathrm{H}\right\}$ NMR: $\delta$ 213.8, 56.7, 42.0, 36.2, 31.7, 29.5, 28.1, 26.7, 26.6, 24.2.

2-(4-Methoxyphenyl)cyclohexanone (Table 2, entry 6). ${ }^{17}{ }^{1} \mathrm{H}$ NMR: $\delta$ 7.05-7.08 (m, 2 H), 6.86-6.90 (m, 2 H), 3.80 (s, 3 H), 3.56 (dd, $J=5.2,12.4$ Hz, 1 H), 2.40-2.55 (m, 2 H), 2.222.29 (m, $1 \mathrm{H}), 2.10-2.18(\mathrm{~m}, 1 \mathrm{H}), 1.95-2.05(\mathrm{~m}, 1 \mathrm{H}), 1.78-1.88(\mathrm{~m}, 2 \mathrm{H}) .{ }^{13} \mathrm{C}\left\{{ }^{1} \mathrm{H}\right\}$ NMR: $\delta$ $210.9,158.6,131.1,129.6,114.0,56.8,55.4,42.4,35.5,28.1,25.6$.

Spiro[5.5] undecan-1-one (Table 2, entries 7 and 12). 18 White crystals. mp $36-39{ }^{\circ} \mathrm{C}$. ${ }^{1} \mathrm{H}$ NMR: $\delta \quad 2.38(\mathrm{t}, J=6.8 \mathrm{~Hz}, 2 \mathrm{H}), 1.79-1.90(\mathrm{~m}, 4 \mathrm{H}), 1.65-1.74(\mathrm{~m}, 4 \mathrm{H}), 1.45-1.50(\mathrm{~m}, 4$ $\mathrm{H}), 1.29-1.42(\mathrm{~m}, 4 \mathrm{H}) .{ }^{13} \mathrm{C}\left\{{ }^{1} \mathrm{H}\right\} \mathrm{NMR}: \delta$ 217.1, 49.2, 38.8, 33.9, 28.4, 26.5, 22.2, 20.8.

Methyl 3-(2-oxo-cyclohexyl)propionate (Table 2, entry 8). ${ }^{19}{ }^{1} \mathrm{H}$ NMR: $\delta 3.61$ (s, 3 H), 2.21-2.40 (m, 5 H), 1.96-2.01 (m, 3 H), 1.81-1.86 (m, 1 H), 1.58-1.68 (m, 2 H), 1.45-1.54 (m, 1 $\mathrm{H}), 1.34(\mathrm{dq}, J=4.0,12.4 \mathrm{~Hz}, 1 \mathrm{H}) .{ }^{13} \mathrm{C}\left\{{ }^{1} \mathrm{H}\right\} \mathrm{NMR}: \delta 212.7,174.2,51.6,49.9,42.3,34.3,31.8$, 28.2, 25.2, 25.0.

2-(2-Chloroethyl)cyclohexanone (Table 2, entry 9). Pale yellow oil. ${ }^{1} \mathrm{H}$ NMR: $\delta$ $3.59(\mathrm{t}, J=6.4 \mathrm{~Hz}, 2 \mathrm{H}), 2.52-2.60(\mathrm{~m}, 1 \mathrm{H}), 2.21-2.39(\mathrm{~m}, 3 \mathrm{H}), 2.04-2.14(\mathrm{~m}, 2 \mathrm{H}), 1.83-1.88(\mathrm{~m}$, $1 \mathrm{H}), 1.48-1.74(\mathrm{~m}, 3 \mathrm{H}), 1.34(\mathrm{dq}, J=3.6,12.8 \mathrm{~Hz}, 1 \mathrm{H}) .{ }^{13} \mathrm{C}\left\{{ }^{1} \mathrm{H}\right\} \mathrm{NMR}: \delta 212.4,47.6,43.5$, 42.4, 34.3, 32.7, 28.2, 25.4. IR (neat, $\mathrm{cm}^{-1}$ ): 2934, 2861, 1708, 1448. Anal. calcd. (found) for $\mathrm{C}_{8} \mathrm{H}_{13} \mathrm{ClO}: \mathrm{H}, 8.16(8.17) ; \mathrm{C}, 59.81(59.62)$. 
2-Phenoxymethylcyclohexanone (Table 2, entry 10). TLC: $\mathrm{R}_{f}=0.28 .{ }^{1} \mathrm{H}$ NMR: $\delta$ 7.21-7.27 (m, 2 H), 3.93-4.04 (m, 2 H), 2.55-2.63 (m, 1 H), 2.23-2.41 (m, 3 H), 2.13-2.19 (m, 1 H), 2.03-2.08 (m, 1H), 1.83-1.88 (m, $1 \mathrm{H}), 1.58-1.71(\mathrm{~m}, 1 \mathrm{H}), 1.40(\mathrm{dq}, J=4.0,12.4 \mathrm{~Hz}, 1 \mathrm{H})$. ${ }^{13} \mathrm{C}\left\{{ }^{1} \mathrm{H}\right\}$ NMR: $\delta$ 213.0, 159.1, 120.8, 114.7, 65.8, 47.5, 42.5, 34.6, 29.5, 28.3, 25.4. IR (neat, $\mathrm{cm}^{-1}$ ): 2933, 2860, 1708, 1244. Anal. calcd. (found) for $\mathrm{C}_{14} \mathrm{H}_{18} \mathrm{O}_{2}: \mathrm{H}, 8.31(8.10)$; C, 77.03 (76.95).

2-(2-Methoxyethyl)cyclohexanone (Table 2, entry 11). Pale yellow oil. ${ }^{1} \mathrm{H}$ NMR: $\delta$ 3.35-3.42 (m, 2 H), 3.28 (s, 3 H), 2.42-2.50 (m, 1 H), 2.25-2.40 (m, 2 H), 2.01-2.14 (m, 2 H), 1.78$1.87(\mathrm{~m}, 1 \mathrm{H}), 1.61-1.72(\mathrm{~m}, 2 \mathrm{H}), 1.34-1.42(\mathrm{~m}, 2 \mathrm{H}) .{ }^{13} \mathrm{C}\left\{{ }^{1} \mathrm{H}\right\}$ NMR: $\delta$ 213.2, 70.7, 58.7, 47.5, 42.4, 34.5, 29.6, 28.3, 25.3. IR (neat, $\mathrm{cm}^{-1}$ ): 2932, 2861, 1708, 1118. Anal. calcd. (found) for $\mathrm{C}_{9} \mathrm{H}_{16} \mathrm{O}_{2}: \mathrm{H}, 10.32$ (10.21); C, 69.19 (69.28).

\section{References}

1) Hayes, J. F.; Shipman, M.; Twin, H. J. Org. Chem. 2002, 67, 935.

2) Moinet, G.; Brocard, J.; Conia, J. M. Tetrahedron Lett. 1972, 4461.

3) Watanabe, S.; Suga, K.; Fujita, T.; Takahashi, Y. Can. J. Chem. 1972, 50, 2786.

4) Sugawara, M.; Yoshida, J-i. Tetrahedron 2000, 56, 4683.

5) Schmittel, M.; Levis, M. Synlett 1996, 315.

6) Thomas, E. W.; Szmuszkovicz, J. R. J. Org. Chem. 1990, 55, 6054.

7) Mazur, P; Nakanishi, K. J. Org. Chem. 1992, 57, 1047.

8) Kawatsura, M.; Hartwig, J. F. J. Am. Chem. Soc. 1999, 121, 1473.

9) Woods, G. F. U.S. Dept. Com. 1959, 161,508, pp170.

10) Santo, R. P.; Lopes, R. S. C.; Lopes, C. C. Synthesis 2001, 6, 845.

11) (a) Suzuki, M.; Ikeno, T.; Osoda, K.; Narasaka, K.; Suenobu, T.; Fukuzumi, S.; Ishida. A. Bull. Chem. Soc. Jpn 1997, 70, 2269. (b) Frimer, A. A.; Gilinsky-Sharon, P.; Aljadeff, G.; Gottlieb, H. E.; Hameiri-Buch, J.; Marks, V.; Philosof, R.; Rosental, Z. J. Org. Chem. 1989, 54,4653 . 
12) Sisti, A. J.; Rusch, G. M. J. Org. Chem. 1974, 39, 1182.

13) Hegedus, L. S.; Williams, R. E.; McGuire, M. A.; Hayashi, T. J. Am. Chem. Soc. 1980, 102, 497.

14) Mino, T.; Masuda, S.; Nishio, M.; Yamashita, M. J. Org. Chem. 1997, 62, 2633

15) Ranu, B.; Dutta, J.; Guchhait, S-K. Org. Lett. 2001, 3, 2603

16) Bolm, C.; Schlingloff, G.; Weickhardt, K. Angew. Chem. 1994, 106, 1944.

17) Lee, K.; Oh, D.-Y. Tetrahedron Lett. 1988, 29, 2977

18) Sands, R. J. Org. Chem. 1994, 59, 468

19) Molander, G.; Koellner, C. J. Org. Chem. 2000, 65, 8333 